PSICOLOGÍA

IBEROAMERICANA
Psicología Iberoamericana ISSN: 1405-0943

revista.psicologia@ibero.mx

Universidad Iberoamericana, Ciudad de México México

\title{
Creación y validación de una escala de codependencia en el noviazgo para jóvenes
}

Salcedo Callado, Pablo Tonathiu; Rivera Aragón, Sofía

Creación y validación de una escala de codependencia en el noviazgo para jóvenes

Psicología Iberoamericana, vol. 27, núm. 1, 2019

Universidad Iberoamericana, Ciudad de México, México

Disponible en: http://www.redalyc.org/articulo.oa?id=133960951001 


\title{
Creación y validación de una escala de codependencia en el noviazgo para jóvenes
}

\author{
Creation and validation of the codependency scale in dating \\ relationships for young people \\ Pablo Tonathiu Salcedo Callado pablo_tona10@hotmail.com \\ Universidad Nacional Autónoma de México, México \\ http://orcid.org/0000-0003-3093-398X \\ Sofía Rivera Aragón \\ Universidad Nacional Autónoma de México, México \\ http://orcid.org/0000-0003-1170-2430
}

Psicología Iberoamericana, vol. 27, núm. 1,2019

Universidad Iberoamericana, Ciudad de México, México

Recepción: 12 Junio 2019 Aprobación: 19 Septiembre 2019

Redalyc: http://www.redalyc.org/ articulo.oa?id=133960951001
Resumen: La codependencia se asocia comúnmente con adultos casados y los instrumentos para su medición están enfocados en su mayoría para ese grupo de edad y tipo de relación. Sin embargo, se ha encontrado que la codependencia puede surgir también en relaciones prematrimoniales durante la juventud, etapa para la cual no existen escalas específicas que midan este fenómeno con precisión. Por lo tanto, el propósito de esta investigación fue construir una escala de codependencia para jóvenes con relaciones prematrimoniales. Para ello, se condujo un estudio instrumental, con un diseño no experimental en donde participaron 319 jóvenes $(M=20.99$, $D E=1.8)$ con un noviazgo de al menos 1 año de duración. A partir de un análisis factorial exploratorio se encontró una escala con 7 factores: control interpersonal $(\alpha=0.91)$, daño relacional $(\alpha=0.92)$, autosacrificio $(\alpha=0.85)$, orientación rescatadora $(\alpha=0.89)$, dependencia emocional $(\alpha=0.90)$, foco externo de atención $(\alpha=0.82)$ y desgaste $(\alpha=0.83)$, que explican $67.64 \%$ de la varianza. Los resultados permiten observar factores coincidentes con la literatura internacional (control interpersonal, autosacrificio y foco externo de atención) y factores nuevos como efecto de la cultura, tipo de relación y etapa de vida (daño relacional, orientación rescatadora, dependencia emocional y desgaste). Así como una escala válida y confiable para la medición de la codependencia en las relaciones prematrimoniales en la juventud.

Palabras clave: relaciones románticas, psicometría, dependencias afectivas, análisis factorial exploratorio.

Abstract: Codependency is commonly associated with married adults, and instruments of measurement, which focus on a certain age group and type of relationship. However, it has been found that codependency can also arise in premarital relationships during youth, at which stage, there are no specific scales that measure this phenomenon accurately. Therefore, the purpose of this research was to build a codependency scale for young people with premarital relationships. An instrumental study was conducted, with a non-experimental design where they participated 319 young people $(M=20.99$, $\mathrm{SD}=1.8$ ) with a dating relationship of at least one year. Based on an exploratory factor analysis, a scale with 7 factors was found: interpersonal control $(\alpha=.91)$, relational damage $(\alpha=.92)$, self-sacrifice $(\alpha=.85)$, rescue orientation $(\alpha=.89)$, emotional dependence $(\alpha=.90)$, external focus of attention $(\alpha=.82)$ and wear $(\alpha=.83)$, which explain $67.64 \%$ of the variance. The results allow us to observe factors coinciding with the international literature (interpersonal control, self-sacrifice and external focus of attention) and new factors such as the effect of culture, type of relationship and stage of life (relational damage, rescue orientation, emotional dependence and wear), in addition to a valid and reliable scale for measuring codependency in premarital relationships amongst youth. 
Keywords: romantic relationships, psychometry, affective dependencies, exploratory factor analysis.

\section{Introducción}

La codependencia (COD) es un constructo en desarrollo dentro del ámbito científico. Entre sus principales campos de estudio se encuentran las observaciones clínicas (Cermak, 1987; Timmen \& Cermak, 1986), los estudios de género (Cowan \& Warren, 1994) y las investigaciones acerca de la dinámica de pareja (Knudson \& Terrell, 2012). En cada una de estas perspectivas, se han generado medidas y definiciones que han contribuido en la identificación de características para comenzar a definirla con claridad. Un esfuerzo particularmente importante para el avance en la definición de la COD. Fue la investigación de Dear, Roberts y Lange (2005), en la cual se llevó a cabo un análisis de las definiciones más importantes, la que identificó las dimensiones consistentes a través de ellas. A partir de este estudio, se propone la existencia de cuatro factores comunes: control interpersonal, foco externo de atención, autosacrificio y supresión emocional, los cuales han servido como base para el estudio de este fenómeno en la última década.

La mayoría de las investigaciones sobre la COD se han enfocado en población general $\mathrm{o}$ en muestras típicamente codependientes (por ejemplo, individuos autoidentificados o en grupos CoDa [Codependientes anónimos]). En estos grupos, se ha logrado especificar características y correlatos, cada vez con mayor claridad (Marks, Blore, Hine, \& Dear, 2012; Ulusoy \& Güçray, 2017). Sin embargo, también se ha indicado la necesidad de explorar la COD en poblaciones más particulares, en rangos de edad específicos y en relaciones distintas al matrimonio (Chang, 2016; Dear \& Roberts, 2002; Martins-D 'Angelo, Menéndez, Gómez-Benito, \& Silva, 2011; Shou-Fang, Li-Chuan, \& Sieh-Hwa, 2004). Se ha sugerido que la COD en jóvenes en relaciones prematrimoniales resulta importante, ya que los patrones codependientes que se instauran en este grupo de edad suelen repercutir en la capacidad para relacionarse en pareja en etapas de vida posteriores (Holland \& Roisman, 2010; Maleck \& Papp, 2015) lo que podría constituir, en muchas ocasiones, la edad y la relación en donde el patrón de COD aparece.

Así pues, para poder investigar la COD en jóvenes con relaciones prematrimoniales, es preciso contar con instrumentos específicos que puedan evaluar las características de la COD en este grupo de edad y tipo de relación. Para ello, es importante recuperar algunos de los aportes que han generado las escalas más importantes de COD a nivel mundial y que han mostrado hallazgos significativos en función de una medición adecuada de la COD.

Los instrumentos más utilizados para la medición de la COD son la escala Spann-Fischer Codependency Scale (Fischer, Spann, \& Crawford, 1991) y la escala Codependency Assessment Questionnaire (Potter-Efron \& Potter-Efron, 1989). La SF CDS evalúa la COD 
a partir de 16 reactivos distribuidos en cuatro factores (Atención centrada en otros, actividades de cuidado, baja expresión emocional y sentido de propósito derivado de los demás) contando con propiedades psicométricas adecuadas: consistencia interna por Alfa de Cronbach de .86 y confiabilidad Test-Retest de .87 , así como validez de criterio con fenómenos como depresión, ansiedad, estrés y disfunción familiar. Por su parte, el CAQ mide consecuencias afectivas de la COD a partir de 34 reactivos repartidos en ocho factores (confusión, miedo, culpa, desesperación, enojo, negación, rigidez y desarrollo incompleto de la identidad) con índices de consistencia interna de Kuder-Richartson con un valor de .86. Estos instrumentos propiciaron las primeras evaluaciones que hicieron viable la evaluación de la COD tanto en personas típicamente codependientes, como en personas de población general, mostrando que el fenómeno puede estar presente en todo tipo de personas y relaciones. A partir de los hallazgos realizados en estas escalas, se han realizado esfuerzos recientes para medir la COD, adaptados a nuevos modelos teóricos de ella.

El instrumento Holyoake Codependency Index (Dear \& Roberts, 2002) es una medida compuesta por 13 reactivos distribuidos en tres dimensiones (autosacrificio, foco externo de atención y reactividad). Con índices de consistencia interna entre .70 y .85 y una proporción de varianza explicada de $53 \%$, es una medida actual, confiable y válida para el fenómeno en población general y población típica codependiente (Grupos $\mathrm{CoDa}$ ), que además tiene validez concurrente con fenómenos como depresión, ansiedad, estrés, autoestima y baja expresión emocional (Dear \& Roberts, 2002). A diferencia de sus predecesoras, la escala HCI se construyó considerando reactivos que no pudiesen confundirse con otros fenómenos, tales como la sumisión o el trastorno de personalidad borderline (Marks et al., 2012) e intentando representar un modelo de COD que unificaba las dimensiones más importantes encontradas hasta esa fecha (Dear et al., 2005). Sin embargo y pese a su utilidad, los autores de la escala refieren algunas limitaciones: la HCI mide solo tres aspectos (autosacrificio, foco externo de atención y reactividad) de los cuatro propuestos por su modelo de medida, por lo que su representación de las dimensiones teóricas de las que parte es parcial. De ello, los autores sugieren realizar estudios que intenten representar totalmente las dimensiones del modelo de medida.

Para responder a las limitaciones referidas por los autores del HCI, Marks et al. (2012) produjeron la escala compuesta de codependencia (CCS, por sus siglas en inglés). Esta escala de 19 reactivos se basa en el mismo modelo de medida que el HCI e intenta representar la totalidad de las cuatro dimensiones del fenómeno propuestas por Dear et al. (2005). No obstante, si bien logran encontrar una escala válida y confiable por sus propiedades psicométricas adecuadas (consistencia interna entre .80 y .85 y $45 \%$ de la varianza explicada), los autores refieren que no se logra representar nuevamente una de las dimensiones propuestas por la teoría original; en este caso, supresión emocional; y sugieren, por primera ocasión, que es posible que la variación en los factores representados 
al tomar este modelo de medida, pueda deberse a las variaciones en los momentos de medición, contextos geográficos, culturas y tipos de poblaciones. Esta sugerencia se ve reforzada en estudios de validación de la escala en otras culturas (Turquía), en donde también existe una variación en los factores que la conforman basándose en el mismo modelo de medida (Ulusoy \& Güçray, 2017).

La sugerencia de la sensibilidad de los factores de la COD propuesta en el estudio de Marks et al. (2012) tiene como evidencia adicional las escalas producidas en Latinoamérica. Los instrumentos de COD en esta región [por ejemplo, la Escala de Codependencia Global (Zarco, 2005); la Escala Argentina de Codependencia (Biscarra, Brandariz, Lichtenberger, Pletzer, \& Cremonte, 2013); el Instrumento de Codependencia (ICOD) (Noriega \& Ramos, 2002)] tienen estructuras factoriales que muestran dichas variaciones. Como resultado, estas escalas presentan estructuras factoriales diversas, exponiendo que la COD en esta zona se presenta de manera diferente e integra en su estructura elementos de la cultura en donde se investigue.

En particular, el ICOD (Noriega \& Ramos, 2002) es considerado como uno de los instrumentos de COD más utilizados de Latinoamérica. Construida en México, esta escala cuenta con 30 reactivos en 4 dimensiones (mecanismo de negación, desarrollo incompleto de la identidad, represión emocional y orientación rescatadora) con un Alfa de Cronbach de .90 y $50.8 \%$ de la varianza explicada, siendo una escala válida y confiable. Uno de sus aportes principales es generar una evaluación de la COD para una población con características particulares (esposas de personas que padecen alcoholismo). Con esto, se fundamenta la necesidad de producir evaluaciones específicas para este fenómeno, que sirvan para medirlo en diferentes poblaciones presentes en la sociocultura mexicana.

Gracias a los aportes de las escalas más importantes y las escalas presentes en Latinoamérica, es posible identificar necesidades para la creación de instrumentos de COD adecuados a un contexto y población específica. Es preciso partir de un modelo de medida actualizado que proporcione elementos generales y aceptados, por lo cual se toma el modelo de Dear et al. (2005), que también es base de las escalas HCI y CCS. También es necesario identificar los elementos de la COD que pueden variar en el contexto cultural mexicano, para ello se consideran los indicadores de la escala ICOD, así como se retoman los resultados de un estudio exploratorio cualitativo sobre la COD en jóvenes mexicanos (Salcedo, Rivera, \& Reyes-Lagunes, 2016). Finalmente, es necesario brindar evidencia sobre la validez y confiabilidad de la escala creada, para que se provea de seguridad para su uso en futuras investigaciones. Con lo cual, el propósito de esta investigación fue la construcción y validación de una escala de COD que permita evaluarla en jóvenes con relaciones prematrimoniales de forma que se puedan identificar estos patrones con prontitud. 


\section{Método}

El presente estudio tuvo como pregunta de investigación: ¿cuáles son las dimensiones de la COD en relaciones prematrimoniales durante la juventud? Con la finalidad de responder dicha pregunta, se procedió a un estudio instrumental (Carretero-Dios \& Pérez, 2005) con un diseño no experimental. Este tipo de estudios están enfocados en el desarrollo y validación de instrumentos de medición, a través del análisis de los reactivos y las dimensiones latentes que subyacen a ellos. Para este estudio, la técnica elegida para la exploración de estas dimensiones fue el análisis factorial exploratorio (AFE) a través de la extracción por ejes principales. Esta técnica ha mostrado previamente su capacidad para la exploración de dimensiones latentes a través de indicadores observados, y es la indicada a realizar en estudios que se enfocan en el desarrollo de instrumentos (Costello \& Osborn, 2005).

\section{Participantes}

En esta investigación participaron anónima y voluntariamente 319 jóvenes (37.2\% hombres, $62.5 \%$ mujeres, $0.3 \%$ no lo mencionaron) considerando como criterio para el tamaño de la muestra tener al menos cinco personas por cada reactivo a analizar (Nunnally $\&$ Berstein, 1994). Los datos de los participantes fueron recopilados en distintas zonas geográficas (por ejemplo, plazas comerciales, parques, alamedas, etcétera.) de la Ciudad de México entre los meses de agosto a diciembre de 2017. El método de muestreo fue no probabilístico (accidental), solicitando la participación de manera individual o grupal. La edad de los participantes osciló entre los 18 y 24 años $(\mathrm{M}=20.99, \mathrm{DE}=1.8)$. Se incluyó a participantes que estuvieran en una relación de noviazgo de al menos un año de duración (80.9\%) o que hubieran experimentado una relación de noviazgo de al menos un año en algún momento previo (19.1\%). El rango de duración de las relaciones fue de 12 hasta 102 meses. Finalmente, se excluyó a las personas que estuviesen casados, vivieran junto con su pareja o tuvieran hijos, debido a que son condiciones que pueden modificar la vivencia y el compromiso de una relación prematrimonial.

\section{Instrumentos}

Se presentó a los participantes un cuestionario compuesto por una serie de preguntas sociodemográficas (por ejemplo, sexo, edad, estatus de la relación, tiempo de duración de la relación, etcétera.) y la primera versión de la Escala de Codependencia en el Noviazgo (ECDN) en la cual se incluyó un apartado de instrucciones en donde se solicitaba a los participantes que pensaran en su relación de noviazgo o su última relación de noviazgo para responder a cada una de las afirmaciones. Los 63 reactivos que componían la escala fueron aleatorizados para 
su presentación en el cuestionario y se colocaron con un formato de respuesta tipo Likert de cinco puntos ( $1=$ Totalmente en desacuerdo a $5=$ Totalmente de acuerdo). Las categorías de los reactivos, su correspondencia con el modelo de medida o el estudio exploratorio cualitativo y un ejemplo de ellos, se detallan en la Tabla 1.

Tabla 1

Distribución de los reactivos en las categorías del modelo de medida o estudio cualitativo

\begin{tabular}{|c|c|c|c|}
\hline $\begin{array}{l}\text { Categoría de los } \\
\text { reactivos en } \\
\text { primera versión de } \\
\text { la escala }\end{array}$ & $\begin{array}{l}\text { Correspondencia con } \\
\text { el modelo de medida } \\
\text { o el estudio } \\
\text { cualitativo }\end{array}$ & $\begin{array}{l}\text { Número de } \\
\text { reactivos }\end{array}$ & Ejemplos de reactivos \\
\hline $\begin{array}{l}\text { Control } \\
\text { interpersonal }\end{array}$ & Modelo de medida & 11 & $\begin{array}{l}\text { Me gusta saber dónde está mi } \\
\text { novio(a) todo el tiempo. }\end{array}$ \\
\hline Autosacrificio & Modelo de medida & 11 & $\begin{array}{l}\text { Las necesidades de mi novio(a) } \\
\text { van primero que las mías. }\end{array}$ \\
\hline $\begin{array}{l}\text { Foco externo de } \\
\text { atención }\end{array}$ & Modelo de medida & 11 & $\begin{array}{l}\text { No puedo dejar de pensar en mi } \\
\text { novio(a). }\end{array}$ \\
\hline $\begin{array}{l}\text { Supresión } \\
\text { emocional }\end{array}$ & Modelo de medida & 11 & $\begin{array}{l}\text { Mi relación me provoca cosas que } \\
\text { no debo expresar. }\end{array}$ \\
\hline $\begin{array}{l}\text { Daño en la } \\
\text { relación }\end{array}$ & Estudio cualitativo & 10 & Creo que mi relación es dañina. \\
\hline $\begin{array}{l}\text { Orientación de } \\
\text { rescate }\end{array}$ & Estudio cualitativo & 9 & $\begin{array}{l}\text { Soy responsable de los problemas } \\
\text { de mi novio. }\end{array}$ \\
\hline
\end{tabular}

\section{Procedimiento}

\section{Construcción del instrumento}

Para la redacción de los reactivos que compondrían la primera versión de la ECDN se partió de la revisión de los componentes de la COD propuestos en el modelo de medida de Dear et al. (2005), las dimensiones obtenidas en el instrumento ICOD y los indicadores de un estudio exploratorio cualitativo en el que se indagó cuál era la definición de la COD en jóvenes mexicanos (Salcedo et al., 2016). Los reactivos redactados, tomando como base el modelo de medida, se realizaron identificando los comportamientos, sentimientos y pensamientos descritos para cada uno de los componentes de la COD: control interpersonal, autosacrificio, supresión emocional y foco externo de atención. Mientras que para la redacción de los reactivos provenientes del estudio exploratorio cualitativo y la escala ICOD, se tomó como indicadores las definidoras obtenidas de las redes semánticas que se 
realizaron en el estudio cualitativo y las dimensiones planteadas en el instrumento ICOD, agrupándolas para esta investigación en dos grupos de comportamientos: daño en la relación y orientación de rescate. De este modo se obtuvieron los 63 reactivos que conformaron la versión inicial de la escala.

\section{Aplicación del instrumento}

La aplicación del instrumento se realizó de manera individual y grupal en diferentes zonas geográficas de la Ciudad de México, acercándose únicamente a los participantes que no se encontraban haciendo alguna otra actividad. Una vez que se les explicaba el propósito de la investigación y las características necesarias para formar parte de él, se les preguntaba "si estarían interesados en participar" y se procedía a darles el cuestionario comentando que "se sintieran libres de responder, ya que nadie conocería ninguna de sus respuestas ni podría identificarlos”. Posteriormente, se describió el instrumento y se solicitó a los participantes que se encontraban en grupo se separaran unos de otros para garantizar una respuesta autónoma. Finalmente, se pidió amablemente que no dejaran ninguna afirmación sin respuesta y el aplicador se alejó para garantizar una respuesta libre de presión. Al finalizar la aplicación se agradeció a cada persona su participación. El tiempo aproximado de respuesta al cuestionario fue de 25 minutos.

\section{Análisis de Datos}

Los datos fueron analizados con el programa SPSS en su versión 23. Para el desarrollo de la primera versión de la escala, misma que sería puesta a prueba a partir del AFE para encontrar sus dimensiones latentes, se examinó la presencia de sesgo y el poder de discriminación de grupos extremos de cada reactivo a través de análisis de frecuencias y pruebas t de student para muestras independientes. Valores de sesgo superiores a $1 \mathrm{o}$ -1 , en conjunto con un valor de $t$ no significativo se tomaron como criterio para la eliminación de reactivos. Posteriormente, para decidir el tipo de rotación a realizar en el AFE, se analizó una matriz con las correlaciones producto-momento de Pearson entre cada reactivo con el total de los reactivos de la escala (Reyes-Lagunes \& García-Barragán, 2008). La presencia mayoritaria de correlaciones superiores a .4 se tomaron como evidencia a favor de una rotación oblicua o menores a .4 como evidencia a favor de una rotación ortogonal (Costello \& Osborn, 2005). Con ello, se conjuntó una primera versión de la escala, con la que se examinaría la existencia de dimensiones latentes.

Así pues, para la exploración de las dimensiones y como evidencia de validez de constructo, se realizó un AFE con una extracción por ejes principales y rotación oblicua (con normalización Kaiser) en la que no se forzó la conformación de un número de factores específico, considerando solo aquellos con autovalores mayores a 1. Los criterios para 
conservar reactivos en las soluciones factoriales fueron la presencia de comunalidades superiores a 3 en conjunto con pesos factoriales mayores a .4 dentro de un factor y menores a 3 en factores adyacentes, así como un número mínimo de tres reactivos para conformar un factor (Nunnally $\&$ Berstein, 1994). Finalmente, como evidencia de confiabilidad, se realizó la estimación del índice de consistencia interna por Alfa de Cronbach en cada factor de la solución resultante y se consideraron como adecuadas Alfas superiores a .60 (Costello \& Osborn, 2005).

\section{Resultados}

Tras analizar la base de reactivos, se encontró que los 63 reactivos iniciales cumplían con las condiciones de ausencia de sesgo, correcta discriminación de grupos extremos y correlaciones significativas entre cada uno con el total, para ser conservados para su inclusión en el AFE. Se decidió una rotación Oblimin debido a las correlaciones superiores a .4 entre los reactivos. Producto de este análisis, se obtuvo una solución adecuada que convergió en 11 iteraciones, arrojando 39 reactivos distribuidos en siete factores que explican el $67.63 \%$ de la varianza total y un Alfa de Cronbach total de .94. Los datos completos del AFE se pueden observar en la Tabla 2 y los factores y definiciones de los mismos en la Tabla 3. 
Tabla 2

Cargas factoriales y propiedades psicométricas de la escala de codependencia en el noviazgo (ECDN)

\begin{tabular}{|c|c|c|c|c|c|c|c|}
\hline & \multicolumn{7}{|c|}{ Factores } \\
\hline & 1 & 2 & 3 & 4 & 5 & 6 & 7 \\
\hline $\begin{array}{l}\text { 12.-Me molesta que mi novio(a) hable con amigos } \\
\text { del sexo opuesto. }\end{array}$ & .75 & .01 & -.02 & .02 & -.05 & .08 & .02 \\
\hline $\begin{array}{l}\text { 9.-Quiero que mi novio(a) no conviva con personas } \\
\text { que yo no conozco. }\end{array}$ & .74 & .03 & -.09 & -.15 & .06 & -.03 & .07 \\
\hline $\begin{array}{l}\text { 14.-Entre menos amigos del sexo opuesto tenga } \mathrm{mi} \\
\text { novio(a) es mejor. }\end{array}$ & .71 & .13 & .07 & .07 & -.07 & -.04 & -.10 \\
\hline $\begin{array}{l}\text { 10.-Me gusta saber dónde está mi novio(a) todo el } \\
\text { tiempo. }\end{array}$ & .61 & -.02 & .05 & -.04 & .01 & .20 & -.01 \\
\hline 6.-Siento celos cuando mi novio(a) es insoportable. & .58 & .04 & -.04 & .14 & -.19 & .16 & .06 \\
\hline $\begin{array}{l}\text { 11.-Siento que no estar con mi novio(a) es } \\
\text { insoportable. }\end{array}$ & .54 & .02 & .06 & -.24 & .01 & .18 & -.03 \\
\hline $\begin{array}{l}\text { 17.-Me siento bien cuando puedo controlar todo lo } \\
\text { que hace mi novio(a) en el día. }\end{array}$ & .54 & -.03 & .10 & -.27 & -.01 & -.04 & .07 \\
\hline $\begin{array}{l}\text { 8.-Me siento tranquilo(a) solo si sé todo sobre mi } \\
\text { novio(a). }\end{array}$ & .50 & -.05 & .17 & -.03 & -.01 & .25 & .06 \\
\hline $\begin{array}{l}\text { 39.-Si no estoy con mi novio(a) siento que es } \\
\text { probable que me engañe. }\end{array}$ & .52 & .10 & .05 & .01 & -.25 & -.03 & .15 \\
\hline $\begin{array}{l}\text { 13.-Está mal que mi novio(a) no me cuente todo lo } \\
\text { que le pasa. }\end{array}$ & .48 & -.09 & .19 & -.01 & -.11 & -.02 & .03 \\
\hline 56.-Mi relación me está desgastando poco a poco. & -.06 & .88 & .02 & .03 & .01 & .03 & .01 \\
\hline 53.-Creo que mi relación puede ser poco sana. & .05 & .88 & -.03 & .09 & -.02 & .02 & .03 \\
\hline 54.-La inestabilidad es normal en mi relación. & .04 & .78 & .07 & -.05 & -.04 & -.05 & -.05 \\
\hline $\begin{array}{l}\text { 58.-Creo que mi relación puede estar } \\
\text { destruyéndome. }\end{array}$ & -.07 & .68 & -.04 & -.11 & .01 & .03 & .25 \\
\hline 50.-Creo que mi relación es dañina. & .02 & .60 & .01 & .01 & .01 & .01 & .35 \\
\hline $\begin{array}{l}\text { 52.-Es normal tener muchos problemas con } \mathrm{mi} \\
\text { novio(a). }\end{array}$ & .12 & .52 & .16 & -.13 & -.08 & -.11 & -.03 \\
\hline $\begin{array}{l}\text { 57.-Creo que mi relación puede estar destruyendo a } \\
\text { mi novio(a). }\end{array}$ & -.01 & .51 & -.04 & -.20 & -.01 & .01 & .25 \\
\hline $\begin{array}{l}\text { 33.-Estar siempre a disposición es otra manera de } \\
\text { decir que quiero a mi novio(a). }\end{array}$ & .01 & .12 & .69 & -.01 & -.06 & -.03 & .01 \\
\hline $\begin{array}{l}\text { 31.-Si mi novio me pide ayuda, negarme no es una } \\
\text { opción. }\end{array}$ & .02 & .07 & .66 & .06 & .02 & -.01 & .06 \\
\hline $\begin{array}{l}\text { 32.-Mis decisiones son siempre mejores cuando } \mathrm{mi} \\
\text { novio(a) me ayuda a tomarlas. }\end{array}$ & -.06 & -.01 & .65 & -.14 & .05 & .12 & -.01 \\
\hline $\begin{array}{l}\text { 30.-Me siento bien conmigo si puedo ayudar a mi } \\
\text { novio(a) con todo lo que hace. }\end{array}$ & -.03 & -.06 & .64 & .09 & -.07 & .10 & .06 \\
\hline $\begin{array}{l}\text { 27.-Si mi novio(a) lo necesita puede conocer todo } \\
\text { de mi vida. }\end{array}$ & .04 & -.01 & .59 & .01 & -.11 & .06 & -.02 \\
\hline $\begin{array}{l}\text { 19.-Las necesidades de mi novio(a) van primero que } \\
\text { las mías. }\end{array}$ & .14 & .04 & .55 & -.10 & .03 & .04 & -.01 \\
\hline $\begin{array}{l}\text { 29.-Si mi novio(a) no está de acuerdo, prefiero no } \\
\text { hacer las cosas. }\end{array}$ & .20 & -.02 & .45 & -.26 & .06 & -.01 & .01 \\
\hline $\begin{array}{l}\text { 60.-Soy responsable de los problemas de } \mathrm{mi} \\
\text { novio(a). }\end{array}$ & .03 & .08 & -.01 & -.74 & -.17 & .02 & .01 \\
\hline 61.-Mi novio(a) es responsable de mis problemas. & .10 & .20 & -.10 & -.73 & -.02 & .08 & .06 \\
\hline 62.-Mi novio(a) es responsable de mi felicidad. & -.01 & .04 & .11 & -.65 & -.07 & .16 & -.02 \\
\hline 59.-Soy responsable de la felicidad de mi novio(a). & -.04 & -.03 & .26 & -.57 & -.16 & -.08 & .07 \\
\hline $\begin{array}{l}\text { 46.-Tengo miedo de no poder resolver mis } \\
\text { problemas } \sin \text { mi novio(a). }\end{array}$ & .19 & -.01 & .01 & -.55 & -.10 & .02 & 17 \\
\hline $\begin{array}{l}\text { 41.-Me siento triste al pensar que mi novio(a) no me } \\
\text { necesita. }\end{array}$ & -.05 & -.01 & .01 & -.04 & -.92 & .03 & .01 \\
\hline $\begin{array}{l}\text { 42.-Me siento solo(a) al pensar que mi novio no me } \\
\text { necesita. }\end{array}$ & .07 & .03 & -.09 & -.09 & -.78 & .06 & .05 \\
\hline 40.-Temo que mi novio(a) algún día no me necesite. & .10 & .06 & .12 & -.08 & -.65 & .01 & .03 \\
\hline 1.-Pienso todo el tiempo en mi novio(a). & -.07 & .02 & -.04 & .04 & -.07 & .82 & -.06 \\
\hline 7.-No puedo dejar de pensar en mi novio(a). & .17 & -.05 & .10 & -.03 & .04 & .67 & .13 \\
\hline 2.-Siento que necesito a mi novio(a). & .06 & .02 & .12 & -.05 & -.03 & 61 & -.04 \\
\hline 4.-Necesito pasara mucho tiempo con mi novio(a). & .08 & -.03 & .12 & -.12 & -.04 & .56 & -.07 \\
\hline 44.-Mi relación me hace sentir triste. & .04 & .11 & .03 & .06 & -.04 & -.02 & .83 \\
\hline 45.-Mi relación me hace sentir débil. & .06 & .10 & -.06 & -.08 & .03 & .03 & .80 \\
\hline 43.-Mi relación me hace sentir infeliz. & -.05 & .01 & .08 & -.01 & -.07 & -.06 & .54 \\
\hline $\mathrm{N}^{\circ}$ de reactivos por factor & 10 & 7 & 7 & 5 & 3 & 4 & 3 \\
\hline Porcentaje de varianza explicada & 34.67 & 13.49 & 5.33 & 4.30 & 3.73 & 3.47 & 2.65 \\
\hline Varianza acumulada & 34.67 & 48.16 & 53.49 & 57.79 & 61.51 & 64.99 & 67.64 \\
\hline Alfa de Cronbach & .91 & .92 & .85 & .89 & .90 & .82 & .83 \\
\hline
\end{tabular}


Tabla 3

Nombres y definiciones de los factores de la ECDN encontrados en el análisis factorial exploratorio.

\begin{tabular}{|c|c|}
\hline Factor & Definición \\
\hline 1.- Control interpersonal & $\begin{array}{l}\text { Pensamientos y sentimientos que expresan la necesidad de control } \\
\text { sobre las conductas del novio o novia en función de conservar un } \\
\text { estado de tranquilidad o estabilidad personal }\end{array}$ \\
\hline 2. - Daño relacional & $\begin{array}{l}\text { Pensamientos sobre el impacto negativo que tiene la relación de } \\
C O D \text { en las dos personas involucradas en ella }\end{array}$ \\
\hline 3.- Autosacrificio & $\begin{array}{l}\text { Actitud abnegada ante las peticiones y necesidades de la novia o } \\
\text { novio en detrimento de las propias }\end{array}$ \\
\hline $\begin{array}{l}\text { 4.- Orientación de } \\
\text { rescate }\end{array}$ & $\begin{array}{l}\text { Creencias acerca de la cualidad compartida de los problemas en el } \\
\text { noviazgo, de modo que estos sean solventados y experimentados } \\
\text { por la otra persona como propios }\end{array}$ \\
\hline $\begin{array}{l}\text { 5.- Dependencia } \\
\text { emocional }\end{array}$ & $\begin{array}{l}\text { Repercusión emocional negativa al considerar la posibilidad de no } \\
\text { ser necesitado por la pareja }\end{array}$ \\
\hline $\begin{array}{l}\text { 6. - Foco externo de } \\
\text { atención }\end{array}$ & $\begin{array}{l}\text { Pensamientos y sentimientos excesivos y recurrentes sobre el novio } \\
\text { o novia y la necesidad de estar con él o ella }\end{array}$ \\
\hline 7.- Desgaste & $\begin{array}{l}\text { Diferentes matices de daño emocional que causa la relación de } \\
\text { noviazgo en la persona }\end{array}$ \\
\hline
\end{tabular}

\section{Discusión}

El propósito de esta investigación fue la construcción y validación de una escala de COD para jóvenes con relaciones prematrimoniales. Los resultados muestran un instrumento con dimensiones concordantes con el modelo de medida teórico: control interpersonal, foco externo de atención y autosacrificio (Dear et al., 2005), y dimensiones que proporcionan evidencia de las características del contexto cultural y el tipo de población en la que se enfoca: daño relacional, desgaste, orientación de rescate y dependencia emocional.

La ECDN construida en esta investigación parte de un modelo de medida basado en la propuesta teórica más aceptada para la COD en la actualidad (Dear et al., 2005), producto de ello, incluye los factores: control interpersonal (control constante de los comportamientos de la pareja y sus actividades), autosacrificio (sumisión continua hacia las necesidades de la pareja) y foco externo de atención (atención centrada en las actividades de la pareja). Lo anterior coincide con los hallazgos de Marks et al. (2012) en la escala CCS, en donde se halló una estructura compuesta por estos factores para la población australiana. Esto constituye evidencia sobre la equivalencia entre algunos de los elementos de la COD que han sido encontrados en la población general y típica codependiente y la COD experimentada por los jóvenes con relaciones prematrimoniales. Adicionalmente, este hallazgo refuerza la idea sobre la existencia de algunos elementos básicos de la COD, que se van modificando y enriqueciendo con otras dimensiones que surgen dependiendo de las condiciones socioculturales en donde se investigue (Marks et al., 2012; Martins-D 'Angelo et al., 2011; Ulusoy \& Güçray, 2017). 
Con respecto a la representación del modelo de medida teórico de Dear et al. (2005), al igual que la encontrada en el estudio de Marks et al. (2012), la dimensión teórica, supresión emocional, no se reflejó en los factores que componen a la ECDN. Esto podría indicar que, en el contexto mexicano, las personas que experimentan la COD no suprimen las emociones sino que podrían estar divulgándolas, mostrando una particularidad cultural en la vivencia de este fenómeno.

Al respecto de estas posibles variaciones culturales, la ECDN se construyó considerándolas. Por ello, para la redacción de los reactivos, se tomaron en cuenta los hallazgos obtenidos en un estudio cualitativo realizado anteriormente por Salcedo et al. (2016), así como algunas dimensiones encontradas en el instrumento ICOD. De esta manera, los resultados mostraron factores adicionales a los del modelo de medida teórico: daño relacional (daño que produce la COD y que perciben ambos miembros de la relación), desgaste (matices de daño emocional que causa la relación de $\mathrm{COD}$ en la persona), orientación de rescate (creencias acerca de los problemas en el noviazgo y la necesidad de que sean percibidos por ambos miembros de la pareja) y dependencia emocional (repercusión emocional negativa al considerar la posibilidad de no ser necesitado por la pareja). Estos factores coinciden con dimensiones reportadas con anterioridad en otras escalas latinoamericanas (Biscarra et al., 2013; Noriega \& Ramos, 2002; Zarco, 2005) y enriquecen la estructura de la COD encontrada para los jóvenes en relaciones prematrimoniales. Esto refuerza la evidencia sobre las variaciones de la $\mathrm{COD}$, que pueden no solo estar en función de la cultura, sino también en función del tipo de relación de pareja.

Una contribución importante de la ECDN es la evaluación de la COD en jóvenes con relaciones prematrimoniales. La intención de generar un instrumento para esta población fue la de evaluar de manera temprana la existencia de la COD y la severidad en cada una de sus dimensiones, para así poder comenzar a explorar y entender el punto de instauración de este patrón de comportamiento para su eventual prevención (Holland \& Roisman, 2010; Maleck \& Papp, 2015). Dados los resultados obtenidos, se puede argumentar que la ECDN es una medida válida y confiable para la tarea de identificación temprana de la COD. Con esto es posible utilizar este instrumento para comenzar a investigar las variables relacionadas a la aparición de este fenómeno en la juventud.

El presente estudio tuvo algunas limitaciones. A partir de las recomendaciones de Nunnally y Berstein (1994) se consideró la conformación de una muestra cuya proporción fuese de al menos cinco personas por cada reactivo para obtener una cantidad de muestra suficiente para la ejecución de un AFE. En futuros estudios se recomienda aumentar la cantidad de la muestra a proporciones mayores (al menos $10 \mathrm{o}$ 15 personas por reactivo) con la intención de encontrar replicabilidad en la estructura factorial de este estudio o confirmarla a través de análisis más rigurosos como el análisis factorial confirmatorio (Costello \& Osborn, 2005). Así mismo, en esta investigación se optó por evaluar las relaciones de noviazgo debido a que son las relaciones prematrimoniales con mayor 
presencia entre los jóvenes dentro de la República Mexicana. Sin embargo, para estudios posteriores se recomienda plantear la validación de la escala para otro tipo de interacción de pareja (por ejemplo, unión libre) o tipos de parejas equivalentes al noviazgo en otras culturas, como las dating relationships en Estados Unidos de América, de modo que se pueda probar la replicabilidad de la estructura factorial encontrada. Finalmente, para este estudio se consideró que la COD es un patrón de comportamiento disruptivo que genera problemas en las personas que lo experimentan (Holland \& Roisman, 2010; Maleck \& Papp, 2015; Noriega \& Ramos, 2002). Sin embargo, sería pertinente evaluar los puntajes bajos y altos de la escala en relación con algunas variables de salud mental (por ejemplo, rumia o regulación emocional) o bienestar general (por ejemplo, afecto negativo y positivo, satisfacción de pareja), de modo que se provea información sobre en qué factores y en qué niveles se encuentran asociaciones con problemas o disrupciones.

\section{Conclusiones}

En conclusión, la ECDN constituye un primer acercamiento a la medición de la COD en grupos de edad y relaciones diferentes a las estudiadas con anterioridad, con lo cual es posible ampliar el campo de conocimiento de este fenómeno y comenzar a estudiar el rango de edad y relación en donde este patrón de comportamiento podría estar surgiendo en las relaciones de pareja. La evidencia encontrada en este estudio es también un avance hacia dilucidar posibles elementos básicos de la COD, que sean consistentes y contrastables a través de las condiciones socioculturales, aunque todavía es necesario ser prudentes y tomar estas posibilidades con precaución.

\section{Agradecimientos}

Se agradece al Consejo Nacional de Ciencia y Tecnología (CONACYT) por la beca otorgada a nombre de Pablo Tonathiu Salcedo Callado, primer autor del artículo con numero de becario 697887 y numero de beca 416141 , con el que se financió la investigación de este.

\section{Referencias}

Biscarra, M. A., Brandariz, R. A., Lichtenberger, A. P., \& Cremonte, M. (2013). Construcción de una Escala de Codependencia. Revista Argentina de Ciencias del Comportamiento, 5(1), 42-51.

Carretero-Dios, H., \& Pérez, C. (2005). Normas para el desarrollo y revisión de estudios instrumentales. International Journal of Clinical and Health Psychology, 5(3), 521-551. http://aepc.es/ijchp/articulos_pdf/ijchp-158 .pdf

Cermak, T. (1987). Diagnostic criteria for codependency. Journal of Psychoactive Drugs, 18(1), 15-20. http://dx.doi.org/10.1080/02791072.1 986.10524475 
Chang, S. H. (2016). Testing a model of codependency for college students in Taiwan based on Bowen's concept of differentiation. International Journal of Psychology, 53(2), 107-116. https://doi.org/10.1002/ijop.1227 1

Costello, A. B., \& Osborne, J. W. (2005). Best practices in exploratory factor analysis: Four recommendations for getting the most from your analysis. Practical Assessment, Research, \& Evaluation, 10(7), 1-9.

Cowan, G., \& Warren, L. (1994). Codependency and gender-stereotyped traits. Journal of Sex Roles, 30(9-10), 631-641. https://doi.org/10.1007/BF015 44667

Dear, G. E., \& Roberts, M. C. (2002). Validation of the Holyoake Codependency Index. The Journal of Psychology, 4(1), 293-314. https://d oi.org/10.3200/JRLP.139.4.293-314

Dear, G. E., Roberts, M. C., \& Lange, L. (2005). Defining codependency: A thematic analysis of published definitions. En S. P. Shohov (Ed.), Advances in psychology research (Vol. 34, pp. 189-205). Hauppauge, NY, US: Nova Science Publishers.

Fischer, J. L., Spann L., \& Crawford, D. (1991). Measuring codependency. Alcoholism Treatment Quarterly, 8(1), 87-100. https://doi.org/10.1300/ J020V08N01_06

Holland, A. S., \& Roisman, G. I. (2010). Adult attachment security and young adults dating relationships over time: Self-reported, observational, and psychological evidence. Developmental Psychology, 46(2), 552-557. http:/ /dx.doi.org/10.1037/a0018542

Knudson, M. T., \& Terrell, K. H. (2012). Codependency, perceived interparental conflict, and substance abuse in the family of origin. The American Journal of Family Therapy, 40(3), 245-257. https://doi.org/10. 1080/01926187.2011.610725

Maleck, S., \& Papp, M. L. (2015). Childhood risky family environments and romantic relationship functioning among young adult dating couples. Journal of Family Issues, 36(5), 567-588. https://doi.org/10.1177/01925 13X13491749

Marks, A. D. G., Blore, R. L., Hine, D. W., \& Dear, G. E. (2012). Development and validation of a revised measure of codependency. Australian Journal of Psychology, 64(3), 119-127. https://doi.org/10.1111/j.1742-9536.201 1.00034.x

Martins-D 'Angelo, R. M., Menéndez, Y., Gómez-Benito, J., \& Silva, Y. F. (2011). Codependencia y sus instrumentos de evaluación: Un estudio documental. Avaliaçao Psicologica, 10(2), 139-150.

Noriega, G., \& Ramos, L. (2002). Construcción y validación del instrumento de codependencia (ICOD) para las mujeres mexicanas. Salud Mental, 25(2), $38-48$.

Nunnally, J. C., \& Bernstein, I. R. (1994). Psychometric theory. Nueva York, NY, US: McGraw-Hill.

Potter-Efron, R. T., \& Potter-Efron, P. (1989). Assessment of co-dependency with individuals from alcoholic and chemically dependent families. Alcoholism Treatment Quarterly, 6(1), 37-57. https://doi.org/10.1300/J0 20V06N01_04

Reyes-Lagunes, I. L., \& García y Barragán, L. F. (2008). Procedimiento de validación psicométrica culturalmente relevante: Un ejemplo. En S. Rivera 
Aragón, R. Díaz Loving, R. Sánchez Aragón \& I. Reyes Lagunes (Eds.), La psicología social en México, Vol. XII (pp. 625 - 636). CDMX, México: AMEPSO.

Salcedo, P., Rivera, S., \& Reyes-Lagunes, I. (2016). La conceptuación de la codependencia del noviazgo. En R. Diaz-Loving, L. I. Reyes-Lagunes, Rivera-Aragon, J. E. Hernandez-Sanchez \& R. García-Falconi (Eds.), Aportaciones actuales de la psicología social (pp. 88-93). México: AMEPSO. ISBN: 978-607-96539-4-1.

Shou-Fang, L., Li-Chuan, W., \& Sieh-Hwa, L. (2004). The study on the relationships between the codependency and trust among high school students. Journal of Educational Psychology, 35(3), 295-318.

Timmen, L., \& Cermak, M. D. (1986). Diagnostic criteria for codependency. Journal of Psychoactive Drugs, 18(1), 15-20. https://doi.org/10.1080/027 91072.1986.10524475

Ulusoy, Y., \& Güçray, S. (2017). Adaptation of composite codependency scale to Turkish: A validity and reliability study. Journal of International Social Research, 10(49), 373-379. https://doi.org/10.17719/jisr.2017.1588

Zarco, I. V. (2005). Relación de la codependencia, el apego, los estilos de amor $y$ los rasgos de masculinidad-feminidad en adultos (Tesis de licenciatura). Universidad Nacional Autónoma de México, CDMX, México. 